Communications in Physics, Vol. 28, No. 2 (2018), pp. 179-187

DOI:10.15625/0868-3166/28/2/10846

\title{
SYNTHESIS OF GOLD NANOBIPYRAMIDS BY SEED-MEDIATED METHOD AND ANTIBACTERIAL ACTIVITIES
}

\author{
NGO VO KE THANH ${ }^{1,2 \dagger}$, HUYNH TRONG PHAT ${ }^{1}$, NGUYEN THI KIM ANH ${ }^{1}$, NGUYEN $^{2}$ \\ DANG GIANG ${ }^{1}$, LAM QUANG VINH ${ }^{3}$, \\ ${ }^{1}$ The Research Laboratories of Saigon High-Tech Park, \\ Saigon Hi-Tech Park Lot I3, N2 Street, Saigon Hi-Tech Park, District 9, Ho Chi Minh City, \\ Vietnam \\ ${ }^{2}$ Institute for Computational Science and Technology, \\ SBI building, Quang Trung Software City, Tan Chanh Hiep ward, district 12, Ho Chi Minh city, \\ Vietnam \\ ${ }^{3}$ Vietnam National University Ho Chi Minh City \\ Community 6, Linh Trung Ward, Thu Duc District, Ho Chi Minh City, Vietnam \\ ${ }^{\dagger}$ E-mail: ngovokethanh@gmail.com; thanh.ngovoke@shtplabs.org
}

Received 1 November 2018

Accepted for publication 31 March 2018

Published 16 June 2018

\begin{abstract}
Metallic nanoparticles as antibacterial agents have been studied for several years. The most used antibacterial nanoparticles are silver nanoparticles. The mechanisms and antibacterial properties of silver nanoparticles are well known, but the effects of gold nanoparticles, especially gold nano bipyramids, are not considered. In this research, we synthesized gold nanobipyramids by seed mediated method using cetyltrimethylammonium bromide surfactant. Gold nanobipyramids was removed cetyltrimethylammonium bromide and modified the surface using polyethylene glycol, polyvinyl alcohol and chitosan as the stabilizers. Besides, antibacterial effects of gold nanobipyramids on both Escherichia coli (Gram negative) and Staphylococcus aureus (Gram positive) were investigated in this paper. The results showed that gold nanobipyramids have good antibacterial activities even at low concentration. The optimal concentration of stabilizers and gold nanobipyramids in antibacterial activities were also studied in this paper.
\end{abstract}

Keywords: gold nanobipyramids, antibacterial activities, nanoparticles, chemical reduction method, seed mediated method.

Classification numbers: 61.46.Df.

(C)2018 Vietnam Academy of Science and Technology 


\section{INTRODUCTION}

Antibiotics that can destroy or slow down the growth of bacteria, are used to treat infections caused by bacteria which may cause illness for human and animals. The first natural antibiotic is penicillin, was discovered by Alexander Flaming in 1928 [1]. Since then, there are many different types of modern antibiotics was discovered, developed and saved millions of lives [2].

Unfortunately, bacterial infections are becoming resistance because of the overusing. Antibiotic resistance is a worldwide problem nowadays. It is found that the resistance has been seen to almost antibiotics which have been developed [3]. According to the European Centre for Disease Prevention and Control (ECDC), an estimated 25,000 people die each year in the European Union from antibiotic-resistant bacterial infections. In 2013, the US Center for Disease Control and Prevention (CDC) estimated at least 2 million people accquire antibiotic resistance and more than 23,000 people die because of antibiotic resistant infections each year in United States [4]. Especially, a new superbug was found that can resist colistin, an antibiotic used as a last resort [5].

Due to the bacteria resist many common antibiotics, infection diseases become one of the problems for human. So that developing the new strategies to treat bacterial infections are researched such as developing new antibiotics, disarming pathogens, starving microbes of nutrients, [6] Among them, metallic nanoparticles are the potential materials as advanced antibacterial agents [7]. Unlike antibiotics, bacteria cannot easily resist against metallic nanoparticles because of their mechanisms. The exact toxic mechanisms of nanoparticles against microorganism are not understood completely [8]. The antibacterial effects based on the interactions between nanoparticles and bacterial cell, including biosorption, uptake cell, nanoparticles aggregation that lead to the membrane damage and toxicity for bacteria [9].

Among inorganic antibacterial nanomaterials, silver nanoparticles are the most used and well known. Besides, there are more types of metallic nanoparticles have been studied such as $\mathrm{TiO} 2, \mathrm{ZnO}, \mathrm{NiO}, \mathrm{CuO}, \mathrm{Cu}[10,12]$. And one of the most noticeable antibacterial agents as well as silver during last year is gold nanoparticles. According to the investigations by Amin et al. [13] and Mubarak Ali et al. [14], silver nanoparticles had higher antibacterial activities than gold nanoparticles. However in our studied, it was found that the antibacterial activities of gold nanobipyramids (NBPs), a rod-like gold nanoparticles, are effective on both Escherichia coli (E. coli) and Staphylococcus aureus (S. aureus).

\section{EXPERIMENTAL}

\section{Preparation of NBPs}

Gold (III) chloride hydrate $\left(\mathrm{HAuCl}_{4} \mathrm{xH}_{2} \mathrm{O}, \sim 52 \%\right.$ Au basis), sodium borohydride $\left(\mathrm{NaBH}_{4}\right.$, 99\%) were purchased from Sigma-Aldrich; cetyltrimethylammonium bromide (CTAB, 99\%), silver nitrate $\left(\mathrm{AgNO}_{3}, 99 \%\right)$ were obtained from Merck; ascorbic acid, sulfuric acid $\left(\mathrm{H}_{2} \mathrm{SO}_{4}, 99 \%\right)$ were purchased from Prolabo Company; polyethyleglycol (PEG, $\mathrm{M}_{n}$ 6000), polyvinyl alcohol (PVA, $\mathrm{M}_{w}$ 31.000-50.000, 98-99\% hydrolyzed), chitosan were provided from Merck. De-ionic (DI) water (18 M $\Omega . c m)$ was used throughout experiments. All chemical materials were GR grade.

In this report, we synthesize NBPs by seed mediated method using ascorbic acid reduction with cetyltrimethylammonium bromide (CTAB) and $\mathrm{AgNO}_{3}$ as capping agents [15]. Firstly, an 50

$\mu \mathrm{l}$ aqueous solution of $10^{-2} \mathrm{M} \mathrm{HAuCl}_{4}$ was mixed with $1 \mathrm{ml}$ solution of $0.2 \mathrm{M} \mathrm{CTAB}$ and $950 \mu \mathrm{l}$ DI water under stirring at room temperature. Then $200 \mu \mathrm{l}$ of cold $\left(4^{\circ} \mathrm{C}\right) 0.1 \mathrm{M} \mathrm{NaBH}_{4}$ solution was 
added into the mixture. The color of solution changed from yellow to brownish. The solution was kept in room temperature at least $24 \mathrm{~h}$ for using as the seed solution. Secondly, the growth solution was made by added $500 \mu \mathrm{l}$ aqueous solution of $10^{-2} \mathrm{M} \mathrm{HAuCl}_{4}$ to $5 \mathrm{ml}$ solution of $0.2 \mathrm{M} \mathrm{CTAB}$ and $4.5 \mathrm{ml}$ DI water under stirring. After that, $37.5 \mu \mathrm{l}$ solution of ascorbic acid (AA) $0.1 \mathrm{M}$ was added. The color of solution immediately changed from brownish yellow to colorless. Next, 50 $\mu \mathrm{l}$ aqueous solution of $0.1 \mathrm{M} \mathrm{AgNO}_{3}$ was dissolved in colorless solution with $\mathrm{pH}$ about 3 (using $\mathrm{H}_{2} \mathrm{SO}_{4} 20 \%$ for adjusting $\mathrm{pH}$ ). Finally, $35 \mu$ l the seeds solution was dropped in the mixture. This solution was kept in room temperature without strirring for $24 \mathrm{~h}$.

\section{Purified and stabilized NBPs}

After prepared, the CTAB layer on NBPs surface is exchanged by biocompatible ligands such as polyethylene glycol (PEG), polyvinyl alcohol (PVA) and chitosan [16]. Under stirring, 10 $\mathrm{ml} \mathrm{NBPs}$ colloids was added to $5 \mathrm{ml}$ biocompatible polymer solution for $2 \mathrm{~h}$ at room temperature. After that, gold nanospheres, free CTAB and polymer molecules were removed using centrifugation at $7000 \mathrm{rpm}$ for $10 \mathrm{~min}$. Then, NBPs were washed 1 time with DI water to remove any residual CTAB and polymer (Fig. 1).

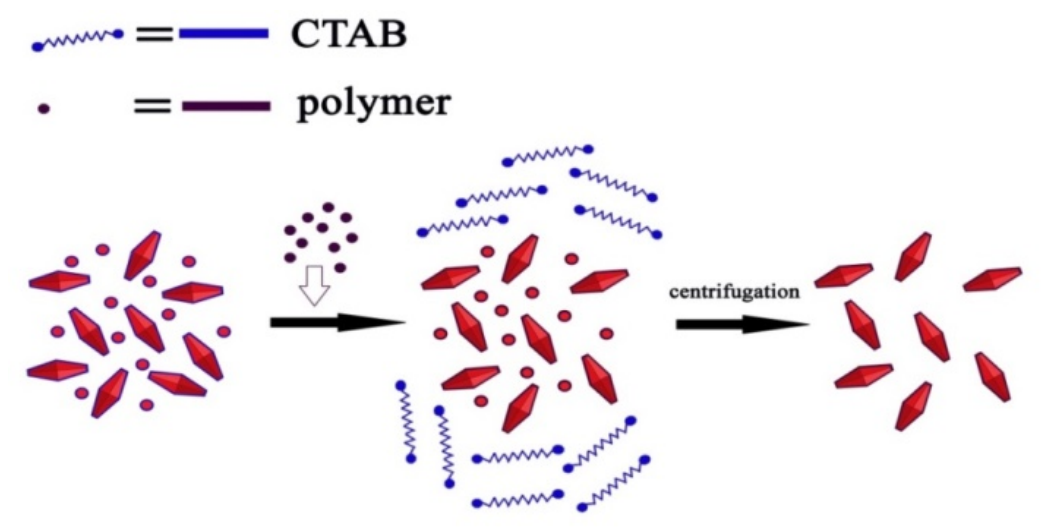

Fig. 1. Process of purification and stabilization of NBPs

\section{Characterization of NBPs}

Ultraviolet-visible (UV-Vis) spectroscopy measurements were carried out on Jasco V-670 to study the aspect ratio (A.R) and purity of NBPs and stabilized NBPs. The spectra of gold colloids were recorded before and after centrifugation. The A.R which is a fraction of length over the diameter of the NBPs particle, can be estimated from the UV-Vis results by Discrete Dipole Approximation (DDA) theory [17] $\left(\lambda_{\max }=96\right.$ A.R + 418). According to the UV-Vis spectrum, the surface plasmon resonance (SPR) of NBPs include two peaks: transverse plasmon resonance (TSPR) peak and longitudinal plasmon resonance (LSPR) peak. The rod-like shape-yield can be estimated based-on the ratio of LSPR/TSPR [18]. The higher LSPR's intensity is, the higher bipyramidal particles is. Otherwise, the higher TSPR peak's intensity is, the higher spherical impurity is.

Transmission electron microscopy (JEM1010-JEOL) was used to determine the size and shape of NBPs. 


\section{Assay for antibacteroal activity of NBPs against $E$. coli and $S$. aureus}

Escherichia coli and Staphylococcus aureus are provided by Pasteur Institute in Ho Chi Minh City. The antibacterial activities of NBPs were evaluated by plate counting method. Added $100 \mu 1$ solution approximately $10^{9}$ colony-forming unit per (CFU/ml) of $E$. coli into $900 \mu 1$ NBPs solution, similar with $10^{7} \mathrm{CFU} / \mathrm{ml}$ of $S$. aureus. Then, $100 \mu 1$ of E. coli-NBPs were inoculated on Eosin methylene blue agar (EMB-Evine) plates or $S$. aureus-NBPs were inoculated on Baird Parker (BP-BK055HA) agar plates. The positive control were used $10^{8}(\mathrm{CFU} / \mathrm{ml})$ of E. coli and $10^{6} \mathrm{CFU} / \mathrm{ml}$ of $S$. aureus. All plates were incubated at $37^{\circ} \mathrm{C}$ for $24 \mathrm{~h}$. Colonies that developed in agar plates were counted after incubation.

\section{RESULTS AND DISCUSSIONS}

\section{Characterization of prepared NBPs and stabilized-NBPs}

The absorption spectra and transmission electron microscopy images of gold nanobipyramids colloids are presented in Fig. 2 and Fig. 3. The UV-Vis results (Fig. 2) show two clearly peaks: one in visible region of TSPR peak and the other in near infrared of LSPR. The max wavelength (LSPR peak) are $753 \mathrm{~nm}$ of NBPs and $749 \mathrm{~nm}$ of stabilized-NBPs after purification. From the LSPR's absorption wavelength, both of the bipyramidal A.R are estimated approximately 3.5 by DDA theory. Besides, the LSPR/TSPR ratio of NBPs before purification is calculated as $2.27 / 2.46$.

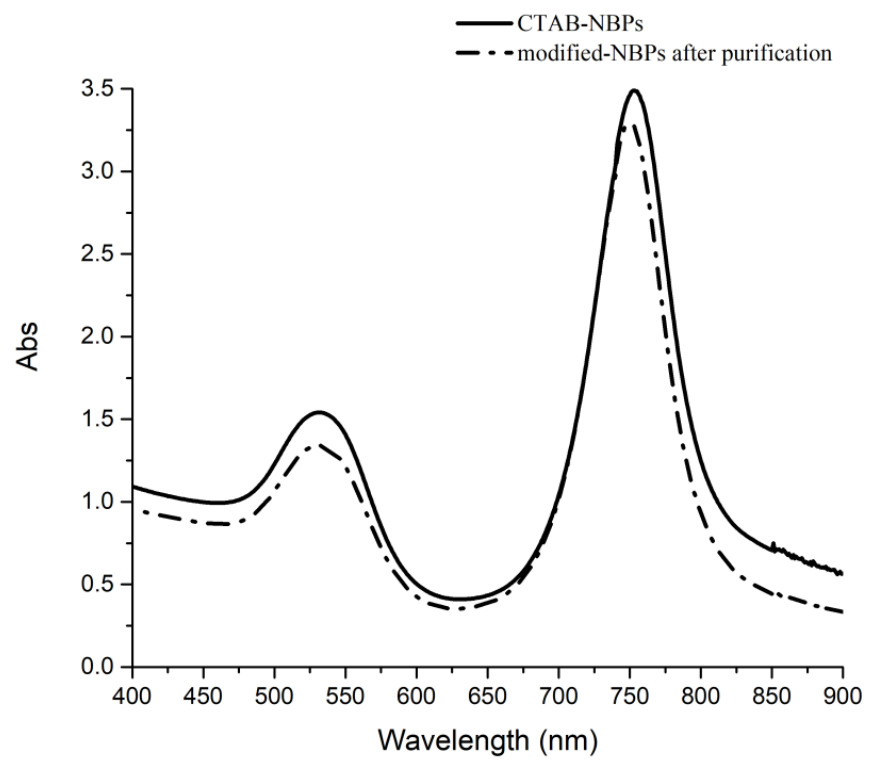

Fig. 2. UV-Vis spectra of prepared NBPs and stabilized NBPs.

According to the UV-Vis results, we can determine that spherical impurities were removed out of stabilized-NBPs colloids without changing shape and size.

TEM images of NBPs were obtained before and after stabilized by PEG, PVA and chitosan. We used ImageJ sofware to measure the length and diameter of NBPs particles from TEM images 
and statistical counting to calculate the average size. NBPs prepared in CTAB surfactant with $78 \pm 6 \mathrm{~nm}$ length and $33 \pm 3 \mathrm{~nm}$ diameter were observed (Fig. 3a). The average size of NPBs particles which re-suspended in PEG, PVA and chitosan-NBPs (Fig. 3b-d) were almost $81 \pm 6 \mathrm{~nm}$ and $33 \pm 3 \mathrm{~nm}$.
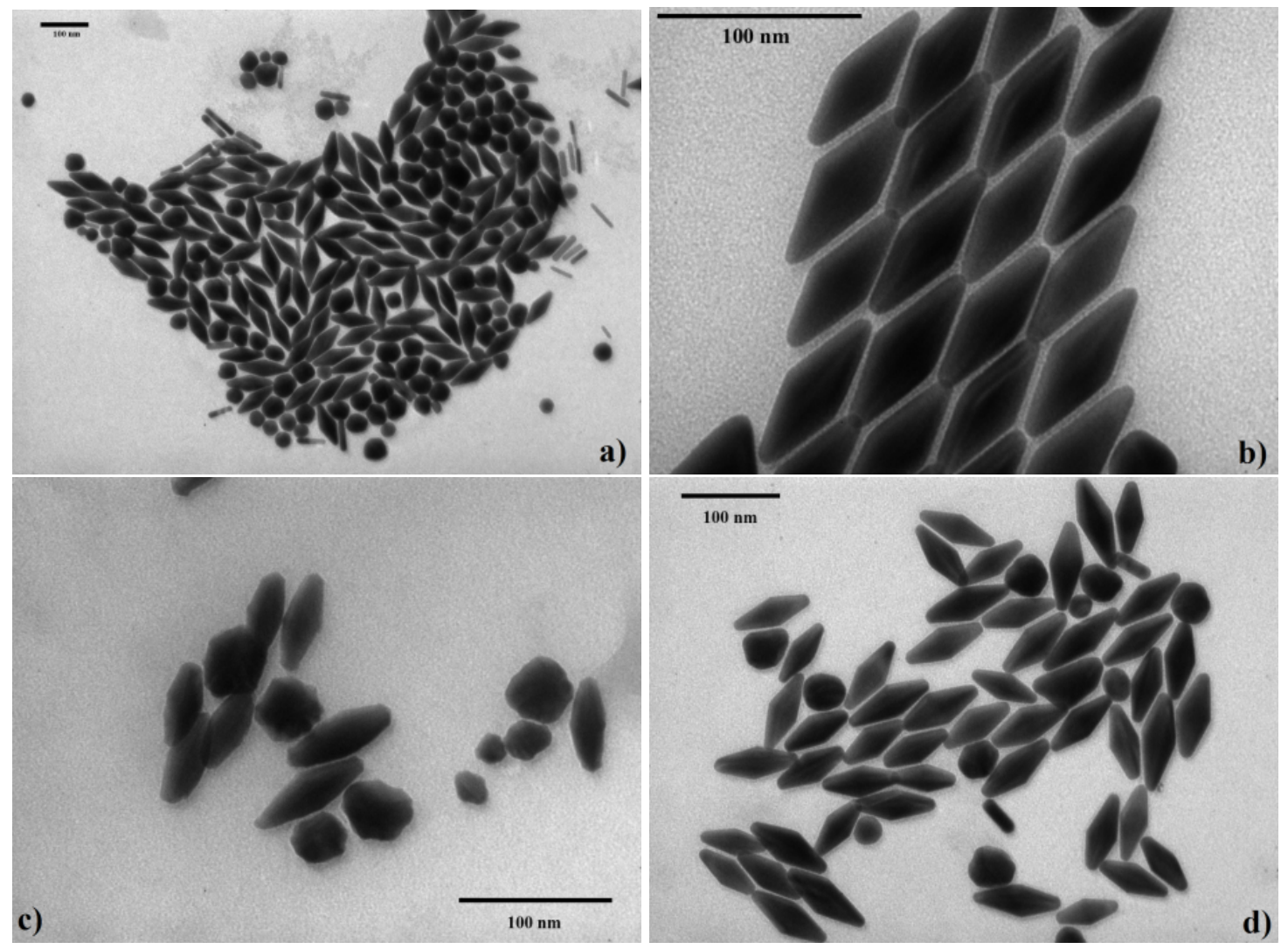

Fig. 3. UV-Vis spectra of prepared NBPs and stabilized NBPs.

\section{III.1. Stability of modified NBPs}

NBPs were removed CTAB by ligand exchange reaction using biocompatible polymer. To investigate the stability of modified NBPs, different polymer concentration from $0.5 \%-2.5 \%$ were used in NBPs suspension as stabilizer. Comparison of SPR of PEG modified and prepared NBPs after 4 weeks was shown in UV-Vis results (Fig. 4a). Inspecting the UV-Vis curve of NBPs at $0.5 \%$ PEG, since the concentration of PEG is so low (0.5\%), not enough to cover NBPs particles, that they were aggregated by time. In higher PEG concentration, it can be seen that the UV curves of PEG-NBPs at $1.0 \%-1.5 \%$ and prepared NBPs are the same, that mean the stability NBPs suspension was also maintained. However, when PEG concentration was increased from $1.5 \%$ to $2.5 \%$, UV-Vis curve changed clearly. The LSPR/TSPR ratio decreases with increasing PEG concentration because of the aggregation of NBPs particles. According to the UV-Vis results, $1.0 \%$ PEG is the optimal concentration stabilizer for NBPs. 


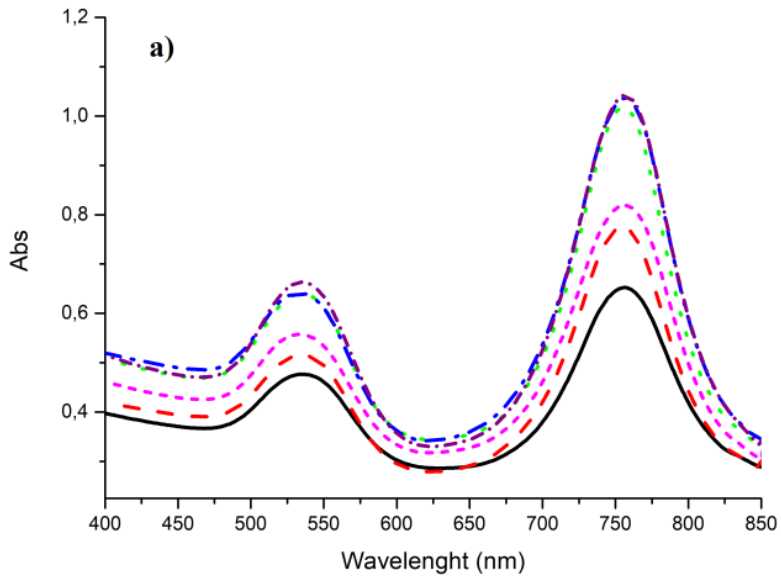

$-2.5 \%$ PEG-NBPs

$-2.0 \%$ PEG-NBPs

- $1.5 \%$ PEG-NBPs

$-\cdot 1.0 \%$ PEG-NBPs

$0.5 \%$ PEG-NBP

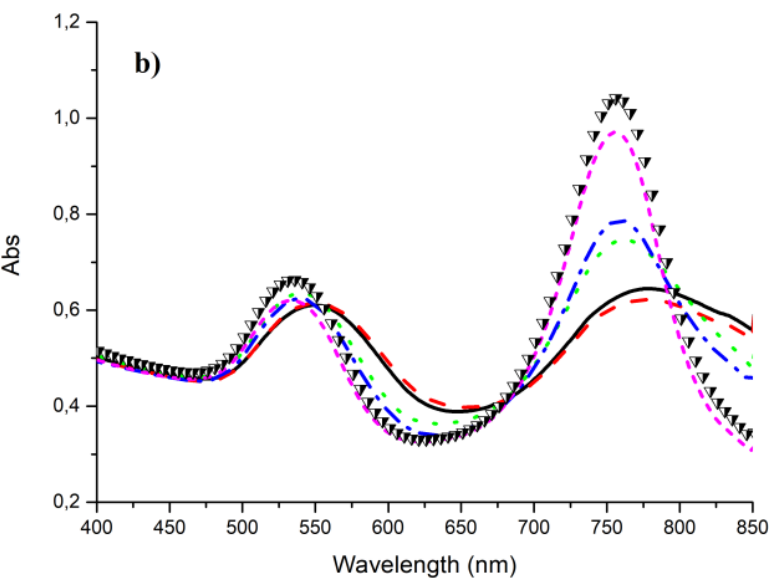

$2.5 \%$ PVA-NBP

$-2.0 \%$ PVA-NBPs

- $1.5 \%$ PVA-NBPs

$-\cdot 1.0 \%$ PVA-NBPs

$\nabla$ CTAB-NBPS

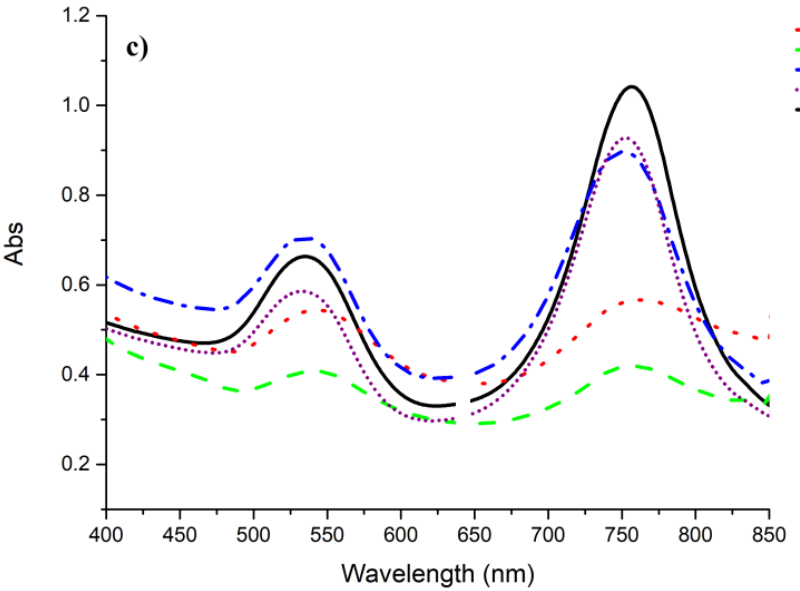

Fig. 4. Stability of modified NBPs in different concentration stabilizers: PEG-NBPs (a); PVA-NBPs (b); chitosan-NBPs (c). 
Fig. $4 \mathrm{~b}$ shows the influence of PVA concentrations to the stability of NBPs. At $0.5 \%$ PVA, the UV-Vis of PVA-NBPs were similar to CTAB-NBPs that approve NPBs suspension is relatively stable after 4 weeks. But the UV-Vis curves changed when PVA concentration increases from $1.0 \%$ to $2.5 \%$. The higher PVA concentration is, the lower LSPR peak's intensity is. From the UV-Vis result, we can conclude $0.5 \%$ PVA is the suitable concentration to stabilize NBPs.

Chitosan stabilized NBPs was presented in Fig. 4c. After 4 weeks, the chitosan-NBPs curves of $0.5 \%$ and $1.0 \%$ concentrations have changed slightly. But it can be seen the chitosanNBPs curves changed clearly with increasing chitosan concentration due to NBPs particles aggregated together.

From the above results, we can realize each polymer and their concentration have influences on the stability of NBPs suspension. If the polymer's concentration is too high, it will be lead to the aggregation of NBPs particles.

\section{Antibacterial activities of stabilized NBPs}
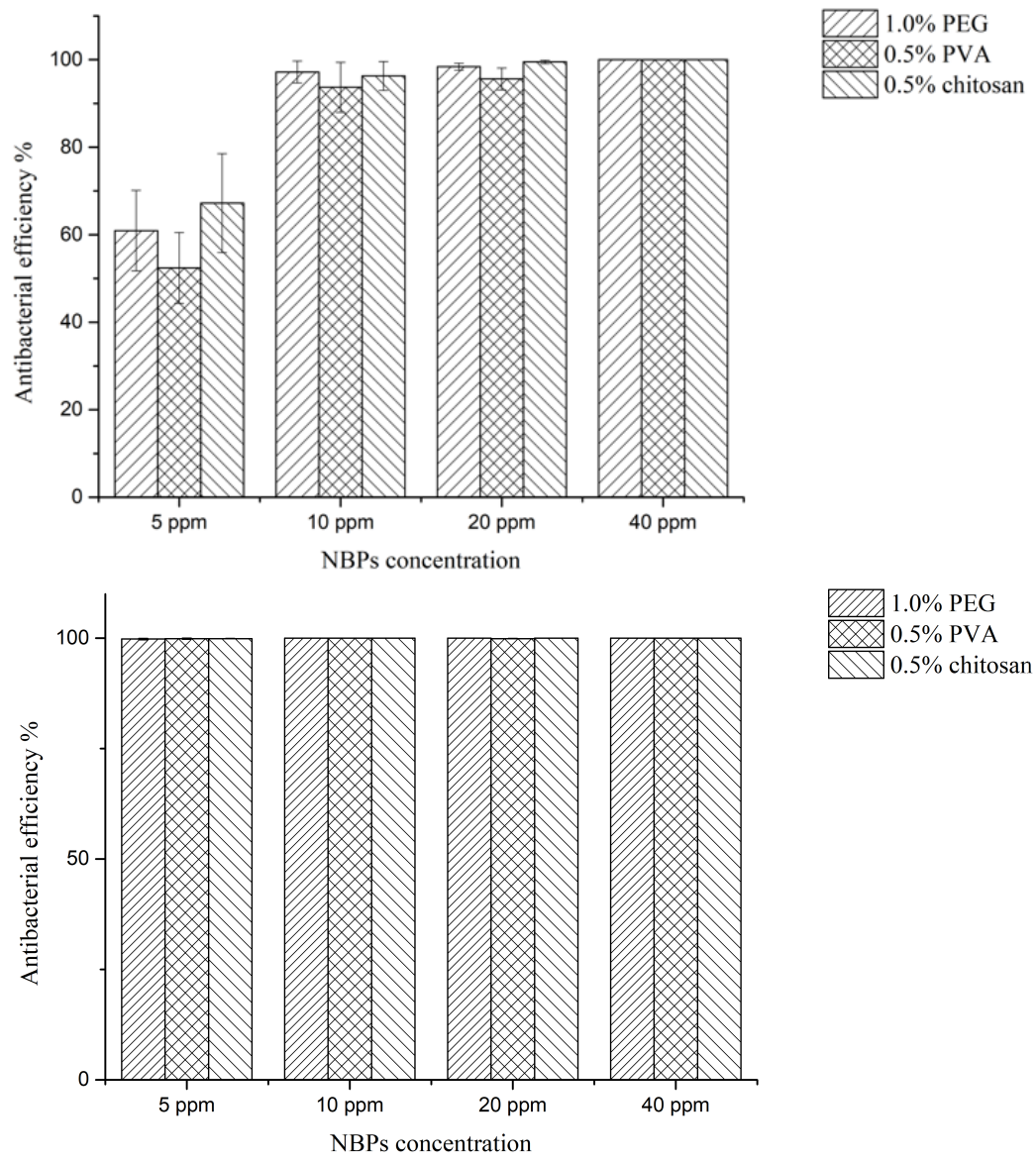

$0.5 \%$ chitosan

Fig. 5. Antibacterial activities of NBPs against E. coli (a) and S. aureus (b). 
Antibacterial tests were performed against $E$. coli and $S$. aureus using different concentrations stabilized NBPs (5 ppm, $10 \mathrm{ppm}$ and $40 \mathrm{ppm})$. Fig. 5a shows the antibacterial efficiencies of stabilized NBPs on E. coli. From the results, it can be seen the killing efficiencies are about $60 \sim 70 \%$ indicated the antibacterial activities were not effective at $5 \mathrm{ppm}$. By increasing the NBPs concentration up to $20 \mathrm{ppm}$, the antibacterial activities are more effective, over $90 \%$ killed bacteria. Comparing with the others, chitosan-NBPs have better killing efficiencies because chitosan also have antibacterial activity $[19,20]$. The antibacterial efficiencies were greatest at $40 \mathrm{ppm}$ on which concentration almost $100 \%$ bacteria cells were killed. Fig. $5 \mathrm{~b}$ shows the antibacterial activities against $S$. aureus. From the results, it can be seen that all of stabilized NBPs at 5 ppm- 40 ppm have $100 \%$ antibacterial efficiencies. Antibacterial tests were performed NBPs is more effective on $S$. aureus than E. coli, even at low concentration of NBPs. The different antibacterial effects are because of the bacteria structure. According to the reports of M. Hajipour et al. [21] and Y. Cui et al. [22], the toxic mechanism of gold nanoparticles against bacteria is two ways: attaching to membrane, decreases the metabolism; permeating and destroying the nucleus of bacteria cell. Gram negative bacteria (E. coli) have an outer wall (lipopolysaccharide layer) as a permeability barrier, therefore NBPs are more difficult to get inside and damage the E. coli bacteria cells than S. aureus.

\section{CONCLUSIONS}

In conclusion, we have demonstrated gold nanobipyramids is a potential material as antibacterial agent. NBPs display their great performance against both Gram positive and Gram negative bacteria at $40 \mathrm{ppm}$. At lower concentration of NBPs, the antibacterial activity on Gram positive is higher than on Gram negative because of the different wall structure of bacteria cells. By changing the surface modification agents using biocompatible polymer, the stability of NBPs was maintained after 4 weeks. Due to antibiotic resistance is a worldwide problem nowadays, gold nanobipyramids with antibacterial properties can be a promising material to develop nanomedicine against the bacterial infections for human in future.

\section{ACKNOWLEDGMENTS}

This research is funded by Laboratories of Saigon Hi-tech Park under Decision 23/QĐTTR\&D. Besides, this work was financially supported by The Institute for Computational Science and Technology, Ho Chi Minh City under Decision 214/QĐ-KHCNTT.

\section{REFERENCES}

[1] Discovery and Development of Penicillin, American Chemical Society (1999).

[2] I. M Gould and A. M. Bal, Virulence 4 (2013) 185.

[3] C. L. Ventola, Pharmacy and Therapeutics 40 (2015) 277.

[4] Antibiotic Resistance Threats In The United States, Centers for Disease Control and Prevention (2013).

[5] P. McGann, E. Snesrud, R. Maybank, B. Corey, A. C. Ong, .R Clifford, M. Hinkle, T. Whitman, E. Lesho and K. E. Schaecher, Antimicrobial Agents and Chemotherapy 60 (2016) 4420.

[6] C. L. Ventola, Pharmacy and Therapeutics 40 (2015) 344.

[7] K. Kon and M. Rai, Journal of Comparative Clinical Pathology 2 (2013) 160.

[8] R. Rawashdeh and Y. Haik, Dynamic Biochemistry, Process Biotechnology and Molecular Biology 3 (2009) 13.

[9] Y. Zhou, Y. Kong, S. Kundu, J. D. Cirillo and H. Liang, Journal of Nanobiotechnology 10 (2012) 19.

[10] K. Adams, D. Y. Lyon, A. McIntosh and P. J. Alvarez, Water Science \& Technology 54 (2006) 327. 
SYNTHESIS OF GOLD NANOBIPYRAMIDS BY SEED-MEDIATED METHOD AND ANTIBACTERIAL ACTIVITIES 187

[11] J. P. Ruparelia, A. K. Chatterjee, S. P. Duttagupta and S. Mukherji, Acta Biomaterialia 4 (2008) 707.

[12] P. Gajjar, B. Pettee, D. W. Britt, W. Huang, W. P. Johnson and A. J. Anderson, Journal of Biological Engineering 3 (2009) 9.

[13] R. M. Amin, M. B. Mohamed, M. A. Ramadan, T. Verwanger and B. Krammer, Nanomedicine 4 (2009) 637.

[14] D. M. Ali, N. Thajuddin, K. Jeganathan and M. Gunasekaran, Colloids and Surfaces B, Biointerfaces 85 (2011) 360.

[15] V. K. T. Ngo, T. P. Huynh, D. G. Nguyen, H. P. U. Nguyen, Q. V. Lam and T. D. Huynh, Advances in Natural Sciences: Nanoscience and Nanotechnology 6 (2015) 035016

[16] .F Ramanery, A. Mansur, H. Mansur, S. Carvalho and M. Fonseca, Nanoscale Research Letters 11 ( 2016) 187.

[17] D. Chateau, A. Liotta, F. Vadcard, J. R. G. Navarro, .F Chaput and J. Lermé, Nanoscale Research Letters 7 (2015) 1934

[18] L. Vigderman and ER Zubarev, Chemistry of Materials 25 (2013) 1450.

[19] R. C. Goy, D. Britto and O. Assis, Polímeros 19 (2009) 241.

[20] L. Qi, Z. Xu, X. Jiang, C. Hu and X. Zou, Carbohydrate Research 339 (2004) 2693.

[21] M. J. Hajipour, K. M. Fromm, A. A. Ashkarran, D. J. Aberasturi, I. R. Larramendi, T. Rojo, V. Serpooshan, W. J. Parak and M. Mahmoudi, Trends in Biotechnology 30 (2012) 499.

[22] Y. Cui, Y. Zhao, Y. Tian, W. Zhang, X. Lü and X. Jiang, Biomaterials 33 (2012) 2327. 
